\title{
CANCER
}

\section{Diffuse idiopathic pulmonary neuroendocrine cell hyperplasia: an under-recognised spectrum of disease}

\author{
Susan J Davies, John R Gosney, David M Hansell, Athol U Wells, Roland M du Bois, Margaret \\ M Burke, Mary N Sheppard, Andrew G Nicholson
}

Thorax 2007;62:248-252. doi: 10.1136/thx.2006.063065

See end of article for authors' affiliations

\section{Correspondence to:} Professor A G Nicholson, Department of Histopathology, Royal Brompton Hospital, Sydney Street, London SW3 6NP,

UK; a.nicholson@rbht.nhs.uk

Received 27 April 2006 Accepted

21 September 2006

Published Online First

10 November 2006
Aims and Methods: A review was undertaken of 19 patients diagnosed with diffuse idiopathic pulmonary neuroendocrine cell hyperplasia (DIPNECH) between 1992 and 2006.

Results: Most patients were women $(n=15)$ and non-smokers $(n=16)$. Clinical presentation was either with symptomatic pulmonary disease (group $1 ; n=9$ ) or as an incidental finding during investigation for another disorder, most frequently malignant disease (group 2; $n=10$ ). In group 1, cough and dyspnoea were the most frequent symptoms, with an average duration of 8.6 years before diagnosis. Both groups showed mainly stable disease without treatment, although one patient progressed to severe airflow obstruction and one was diagnosed at single lung transplantation. Mosaicism with nodule(s) was the typical pattern of DIPNECH on high-resolution computed tomography, but one case had normal imaging despite airflow obstruction. Lung function tests showed obstructive $(n=8)$, mixed $(n=3)$ or normal $(n=5$, all group 2) physiology. Two patients underwent a bronchoalveolar lavage and showed a lymphocytosis $(30 \%)$ with mild chronic bronchiolitis being seen in all biopsies. Tumourlets and associated typical carcinoids $(n=9)$ showed weak positivity for thyroid transcription factor-1. Three patients had atypical carcinoids, one with multiple endocrine neoplasia type 1 syndrome.

Conclusions: DIPNECH is being increasingly recognised, probably because of an increase in the usage and accuracy of investigative imaging and increased awareness of the entity. Most cases remain stable over many years independent of the mode of presentation, although a few patients progress to severe airflow obstruction.
$\mathrm{T}$ he histological appearance of diffuse idiopathic pulmonary neuroendocrine cell hyperplasia (DIPNECH) within the lungs takes many forms. It may comprise a generalised proliferation of scattered neuroendocrine cells, small nodules (neuroendocrine bodies) or a linear proliferation of pulmonary neuroendocrine cells. These proliferations are superficial to the basement membrane and confined to the bronchial and bronchiolar epithelium. When there is extension of neuroendocrine cells beyond the basement membrane, the proliferations are termed tumourlets, which can be localised or diffuse. Nodules $>5 \mathrm{~mm}$ in diameter are classified as carcinoid tumours; they are usually single masses although they can be associated with DIPNECH. Although foci of neuroendocrine cell hyperplasia and tumourlets are often seen in association with other diseases when they are considered to be reactive, ${ }^{1}$ DIPNECH is thought to be a primary proliferation and is sometimes associated with constrictive obliterative bronchiolitis. $^{2}$

DIPNECH was first described in the early 1950 s, ${ }^{3}$ but was not fully recognised and named until 1992. ${ }^{2}$ Although there have been several case reports and two series of six and five patients, respectively, ${ }^{2-7}$ information about the disease is still limited especially with regard to management and prognosis. We have noticed an increase in cases in the past 2 years and therefore sought to review our experience of patients presenting with DIPNECH in order to characterise this disorder further.

\section{METHODS}

Between 1992 and 2006, nine cases were identified in the files of the Royal Brompton and Harefield Hospitals NHS Trust, London, UK. A total of 10 further cases were identified in the personal referral files of two pathologists (AGN/JRG). All cases were reviewed and the diagnosis confirmed using slides stained with H\&E and elastic van Gieson stains. Sections were additionally assessed for the presence of the following histological parameters: neuroendocrine cell hyperplasia, tumourlets, carcinoids, bronchiolar inflammation, constrictive obliterative bronchiolitis, bronchiolar wall thickening, bronchiolectasis and mucus plugging. When slides were available $(\mathrm{n}=10)$, immunohistochemical analysis was performed using antibodies to thyroid transcription factor-1 (TTF-1; Dako, Ely, Cambridgeshire, UK; dilution 1 in 80), CD56 (Novocastra, Newcastle upon Tyne, UK; dilution 1 in 100), CD45 (Dako; dilution 1 in 100), CD20 (Dako; dilution 1 in 400) and CD3 (Dako; dilution 1 in 25).

Lung function measurements were recorded within 6 months of the time of biopsy. Lung function tests at the Royal Brompton and Harefield NHS Trust were measured using an Ohio water-seal spirometer (Ohio Instruments, Atlanta, GA, USA) and measures of gas transfer (diffusing capacity of the lung for carbon monoxide) were made by the single-breath technique using a PK Morgan respirometer (PK Morgan, Chatham, Kent, UK). Results were expressed as percentages of values predicted from the subject's age, sex and height, as described previously. ${ }^{8}$

Thin high-resolution CT (HRCT) sections (1-1.5 mm) at 10 $20 \mathrm{~mm}$ intervals were obtained from all patients. Patients were scanned in a supine position and images were obtained at suspended full inspiration and reconstructed with a high spatial frequency (bone) algorithm and photographed at appropriate window settings (window centre $=-600$ to $-655 \mathrm{HU}$; window width $=1500-1850 \mathrm{HU})$. Imaging was

Abbreviations: DIPNECH, diffuse idiopathic pulmonary neuroendocrine cell hyperplasia; HRCT, high-resolution computed tomography; MEN, multiple endocrine neoplasia 
Table 1 Clinical features of patients with diffuse idiopathic pulmonary neuroendocrine cell hyperplasia

\begin{tabular}{|c|c|c|c|}
\hline Clinical features & $\begin{array}{l}\text { Group } 1(n=9) \\
\text { (Symptomatic) }\end{array}$ & $\begin{array}{l}\text { Group } 2 \text { ( } n=10) \\
\text { (Asymptomatic) }\end{array}$ & Overall $(n=19)$ \\
\hline Male : female $(n=19)$ & $2: 7$ & $2: 8$ & $4: 15$ \\
\hline $\begin{array}{l}\text { Smoking history ( } n=17 \text { ) } \\
\text { (never: ex-smoker : current) }\end{array}$ & $5: 2: 1$ & $7: 2: 0$ & $12: 4: 1$ \\
\hline Mean age at onset (years) & $49.1(31-67)$ & NA & \\
\hline \multicolumn{4}{|l|}{$\begin{array}{l}\text { Presenting complaints: } \\
\text { Pro }\end{array}$} \\
\hline Cough & $4 / 9$ & $0 / 10$ & $4 / 19$ \\
\hline Increasing dyspnoea & $6 / 9$ & $0 / 10$ & $6 / 19$ \\
\hline Pleuritic chest pain & $2 / 9$ & $0 / 10$ & $2 / 19$ \\
\hline Haemoptysis & $1 / 9$ & $1 / 10^{*}$ & $2 / 19$ \\
\hline Asymptomatic & $0 / 9$ & $9 / 10^{*}$ & $9 / 19^{*}$ \\
\hline Previous malignancy & $0 / 9$ & $8 / 10$ & $8 / 19$ \\
\hline History of asthma & $3 / 9$ & $2 / 10$ & $5 / 19$ \\
\hline $\begin{array}{l}\text { Lung function ( } n=16 \text {; } \\
\text { obstructive : mixed : normal) }\end{array}$ & $5: 3: 0$ & 3: $0: 5$ & $8: 3: 5$ \\
\hline $\begin{array}{l}\text { Mean duration of illness before } \\
\text { diagnosis (years) }\end{array}$ & 8.6 & NA & 8.6 \\
\hline Bronchoalveolar lavage & Lymphocytosis $2 / 2$ & - & Lymphocytosis $2 / 2$ \\
\hline \multicolumn{4}{|l|}{ Treatment† } \\
\hline Steroids & $2 / 6$ & $0 / 8$ & $2 / 14$ \\
\hline Watch and wait & $3 / 6$ & $4 / 8$ & $7 / 14$ \\
\hline \multicolumn{4}{|l|}{ Follow-up } \\
\hline $\begin{array}{l}\text { Clinically stable (alive } \\
\text { with disease) }\end{array}$ & $\begin{array}{l}5 / 7 \text { (range } 1-12 \text { years, } \\
\text { mean } 5.8 \text { years) }\end{array}$ & $\begin{array}{l}6 / 7 \text { (range } 0.2-2.2 \text { years, } \\
\text { mean } 1.0 \text { year) }\end{array}$ & $11 / 14$ \\
\hline Clinical deterioration & $1 / 7$ & $0 / 7$ & $1 / 14$ \\
\hline Died of DIPNECH & $1 / 7 \ddagger$ & $0 / 7$ & $0 / 14$ \\
\hline Died of other disease & $0 / 7$ & $1 / 7 \S$ & $1 / 14$ \\
\hline
\end{tabular}

DIPNECH, diffuse idiopathic pulmonary neuroendocrine cell hyperplasia.

$\mathrm{n}=$ number of patients for whom data were available.

*Symptoms felt due to other disease rather than DIPNECH.

tDoes not include immunosuppression after transplantation $(n=1$, group 1$)$, chemotherapy for carcinoma $(n=2$, group

2) and chemotherapy for metastatic atypical carcinoid ( $n=1$, group 2).

¥One patient died of chronic rejection after transplantation.

$\S$ One patient died of carcinoma of the large bowel at 5 years.

reviewed by one radiologist with an interest in thoracic disease (DMH) and was assessed for the following features: groundglass attenuation, nodules, mosaic attenuation pattern, bronchiectasis, bronchial wall thickening and other parenchymal disease. Clinical data were obtained from the clinical case notes and referral letters to pathologists. The study was approved by the Brompton, Harefield and NHLI ethics committee.

\section{RESULTS}

\section{Clinical data}

There were 15 women and 4 men. Smoking history was available on 17 patients, 12 of whom were never-smokers, 4 were ex-smokers and 1 was a current smoker. Age at diagnosis ranged from 46-78 (mean 57.5) years Analysis of other clinical data was undertaken through division into two groups (table 1), those who presented with respiratory symptoms ascribable to DIPNECH (group 1) and those in whom DIPNECH was picked up incidentally during clinical investigations for other diseases (group 2). In group $\mathrm{l}(\mathrm{n}=9)$, age at presentation with symptoms ranged from 31 to 67 (mean 49.1) years. In all, seven patients were women and five were non-smokers. Two patients were ex-smokers, one was a current smoker and the smoking history was not known in one patient. Presentation was typically with non-productive cough $(\mathrm{n}=4)$ and/or dyspnoea $(n=6)$; these symptoms were present for 4-16 (mean 8.6) years before correct diagnosis. Two patients had chest pain and one patient had haemoptysis. Three patients had been diagnosed with asthma. In one of these patients an acute exacerbation prompted a chest radiograph that showed a coin lesion in the right middle lobe, interpreted clinically as a tumour (table 1).

In group $2(\mathrm{n}=10)$, nine were asymptomatic. One patient had haemoptysis that on review was thought to be due to coexistent metastatic carcinoma of the large bowel. Eight patients were women and seven were never smokers. Two were ex-smokers and the smoking history was not known in one patient. Age at diagnosis ranged from 58 to 78 (mean 65.0 ) years. Eight patients had undergone resection for one or more malignancies; one was being investigated after a fall and one after a collapse (table 1).

In group 1, seven patients had chest imaging for review and six of these underwent high-resolution computed tomography (HRCT scanning; table 2). Four HRCT scans showed a mosaic attenuation pattern, reflecting small airways disease, two with multiple associated nodules (fig 1 ), one with a single nodule and one with additional atelectasis. Two patients had bronchial wall thickening and two cases showed cylindrical bronchiectasis. One patient had a normal HRCT scan despite moderate airflow obstruction on lung function tests. One patient had multiple nodules only and one patient had a single nodule on the chest radiograph (HRCT was not performed). Nodules ranged from 2 to $20 \mathrm{~mm}$ in diameter.

In group 2, eight patients had imaging available for review and seven of these had HRCT scans (table 2). Five of these were under surveillance because of a previous malignancy (carcinoma of large bowel $(n=2)$, carcinoma of breast and angiosarcoma of chest wall $(n=1)$, liposarcoma $(n=1)$, gastrinoma in association with multiple endocrine neoplasia (MEN) type 1 syndrome $(\mathrm{n}=1)$ ). All showed multiple nodules ranging from 3 to $15 \mathrm{~mm}$ in diameter. In two other patients, who were investigated after a fall and a collapse, chest radiographs both showed single nodules that were subsequently confirmed on HRCT scans, the nodules measuring $10 \mathrm{~mm}$ and $25 \mathrm{~mm}$, respectively. One patient with multiple nodules also showed mosaicism and airway dilatation. One patient, who was being followed up for evidence of metastatic 


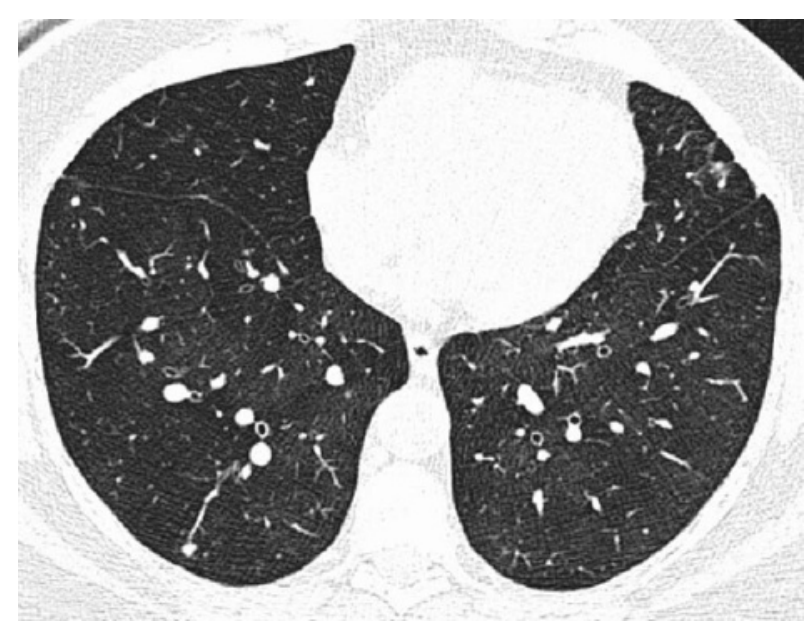

Figure 1 A high-resolution computed tomography scan through the lower lobes shows mosaicism and nodules, a combination of features suggestive of diffuse idiopathic neuroendocrine cell hyperplasia.

disease with a history of previous carcinomas of kidney and breast, had a chest radiograph that showed only multiple nodules. None of the patients in group 2 had bronchial wall thickening. In one patient, follow-up HRCT scans showed new nodules that, due to the rate of growth, were thought to be metastatic carcinoma of the large bowel (the patient died 5 years after presentation).

Lung functions tests (table 1$)$ for group $1(n=8)$ showed moderate to severe airflow obstruction in five patients and a mixed obstructive/restrictive pattern in three patients. In group $2(\mathrm{n}=8)$, five patients had normal lung function whereas three patients had mild to moderate airflow obstruction. Two patients from group 1 underwent bronchoalveolar lavage as part of their investigation. Both showed a lymphocytosis (31\% and 30\%) at presentation, with one patient showing a reduction to $19 \%$ after inhaled steroid therapy.

\section{Histopathology}

All cases showed both neuroendocrine cell hyperplasia and tumourlets to varying degrees involving primarily terminal bronchioles, but also affecting respiratory bronchioles (fig 2). Both were accompanied by varying degrees of fibrosis, more so in relation to tumourlets, and in 13 cases there was constrictive obliterative bronchiolitis (fig 3). The extent of constrictive obliterative bronchiolitis varied from very occasional bronchioles to florid obstruction in the case that eventually required single lung transplantation. Five cases showed focal bronchiolectasis, nine showed mucus plugging (fig 3) and 13 showed thickening of the airway wall itself, features interpreted as secondary to the obstruction of small airways. All cases had a chronic inflammatory cell infiltrate comprising mainly $\mathrm{T}$ cells. Inflammation was mild in intensity in all cases. Occasional foci of follicular hyperplasia were seen in two cases. In the 10 cases where tissue was available for immunohistochemical analysis, CD56 highlighted a greater degree of neuroendocrine cell hyperplasia than was appreciated on H\&E-stained slides and all cases showed weak positive nuclear staining for TTF-1. Nine cases had typical carcinoids (group 1, $\mathrm{n}=4$ and group 2, $\mathrm{n}=5$ ) in association with DIPNECH, all but two showing at least partial spindle cell morphology. One patient with MEN type l syndrome had multiple typical carcinoids and one atypical carcinoid, as defined by World Health Organization criteria. ${ }^{9}$ Two other patients had solitary atypical carcinoids, one with metastasis to mediastinal lymph nodes (table 3).
Table 2 Imaging data on high-resolution computed tomography

\begin{tabular}{llll}
\hline CT findings & Group $^{*}$ & Group 2† & Total \\
\hline Presence of nodules & $4 / 6$ & $7 / 7$ & $10 / 13$ \\
Airway dilatation & $2 / 6$ & $1 / 7$ & $3 / 13$ \\
Bronchial wall thickening & $2 / 6$ & $0 / 7$ & $2 / 13$ \\
Air trapping \pm mosaicism & $4 / 6$ & $1 / 7$ & $5 / 13$ \\
Atelectasis & $1 / 6$ & $0 / 7$ & $1 / 13$ \\
Normal & $1 / 6$ & $0 / 7$ & $1 / 13$ \\
\hline
\end{tabular}

*One other patient had a chest radiograph only, which showed a single nodule.

tOne other patient had a chest radiograph only, which showed multiple nodules.

\section{Management and follow-up}

In group 1, treatment decisions were available for seven patients. One patient treated with inhaled steroids showed deterioration over a period of 4 years, with lung function falling from normal range to a forced expiratory volume in 1 second of $59.8 \%$ predicted and forced vital capacity of $67.5 \%$ predicted. Bronchioalveolar lymphocytosis over this period fell from 31\% to $19 \%$. One patient was treated with oral prednisolone and has remained stable for 6 years. One patient died of chronic rejection 5 years after single lung transplantation. Four patients were managed by observation only, with no active treatment, and had stable disease over $1-12$ years other than a gradual increase in nodule size in one patient. The overall survival was $83 \%$ at 5 years.

In group 2, four patients were managed by observation only and all were alive with stable disease at 6 months-1 year (average 9 months). Two patients are undergoing chemotherapy for carcinoma of the large bowel, one patient is currently undergoing chemotherapy for metastatic atypical carcinoid and one patient died of metastatic carcinoma of the large bowel at 5 years follow-up. One patient is current and has no follow-up.

\section{DISCUSSION}

The first clinical series of six cases describing DIPNECH was published by Aguayo et al in 1992. ${ }^{2}$ These patients were mainly women, had obstructive ( $\mathrm{n}=5$ ) or mixed obstructive/restrictive $(\mathrm{n}=\mathrm{l})$ lung function, showed no clinical association with smoking and showed neuroendocrine cell hyperplasia associated with constrictive obliterative bronchiolitis on histological examination. Follow-up showed that one patient died of progressive disease whereas the remainder had stable disease. Smaller more recent series and case reports have documented similar findings..$^{4-7}$ Our data are therefore similar to those published, but in addition we show that DIPNECH may not be as rare as thought previously and also add new insights into the presentation, clinical behaviour and histological features of this entity.

There seem to be two different modes of clinical presentation for DIPNECH. The first is with symptomatic disease, typically non-productive cough and dyspnoea, which is not necessarily progressive, and an obstructive lung function profile. If an HRCT is undertaken, evidence of small airways obstruction in the form of mosaicism may be seen and the coexistence of multiple pulmonary nodules is a pointer towards the diagnosis of DIPNECH. These observations are similar to those in the one published HRCT series on patients with DIPNECH. ${ }^{4}$

However, one patient had a normal HRCT scan despite moderate airflow obstruction, so the absence of HRCT findings does not negate the diagnosis. Indeed, the fact that symptoms were present for a mean of 8.6 years before diagnosis highlights the non-specific nature of clinical symptoms and reflects the presumption that patients have another indolent or reversible 

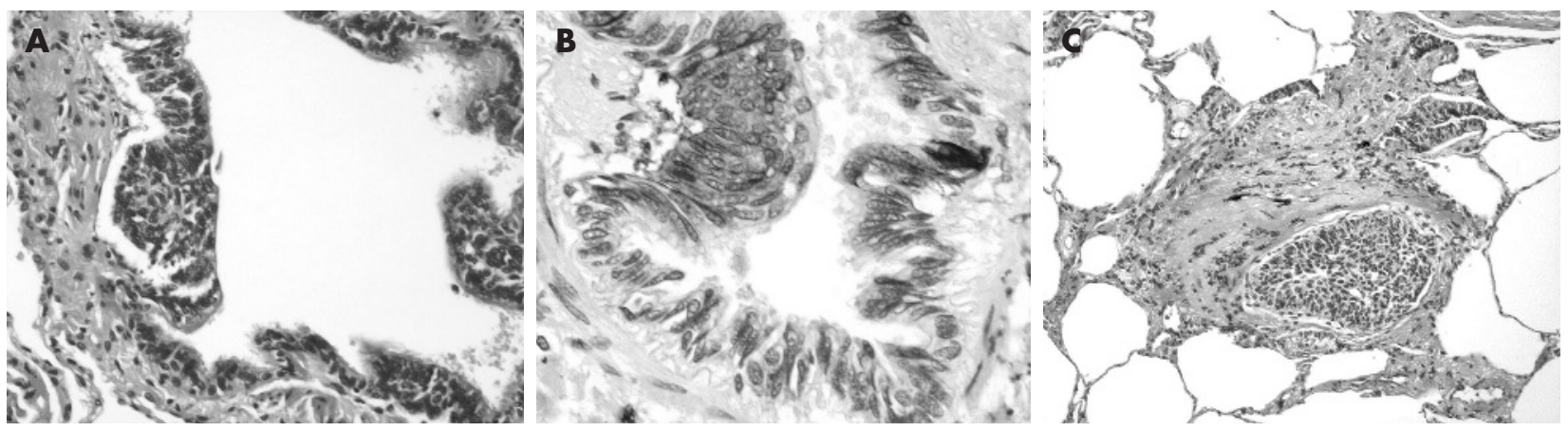

Figure 2 (A) Diffuse idiopathic neuroendocrine cell hyperplasia may comprise an intraepithelial aggregate of neuroendocrine cells, (B) highlighted by staining for the neuroendocrine marker CD56. (C) When neuroendocrine cells extend beyond the basement membrane, the foci are classified as tumourlets.

obstructive airways disease such as asthma (table 1), not necessarily requiring HRCT for diagnosis.

The second mode of presentation is through surgical referral, typically for resection of a pulmonary nodule or nodules that have been found incidentally on imaging performed for reasons unrelated to DIPNECH. Of those in group 2, it is notable that eight patients had a recent history of either carcinoma or sarcoma treated by resection, and nodules were identified either during surveillance for recurrence or during investigation for metastatic spread at the time of presentation. Interestingly, all but one of these cases presented after January 2004, which may reflect the increased usage of HRCT in relation to monitoring for pulmonary metastases. This greater intensity of investigation may be responsible for our increased diagnosis of DIPNECH, and suggests that background neuroendocrine cell hyperplasia is more common than was previously thought. Previous studies looking at the background lung of patients with resected carcinoids show frequent evidence of neuroendocrine cell hyperplasia, ${ }^{10}{ }^{11}$ and it may simply be that the increased use of investigative imaging and biopsy in patients suspected of having recurrent or metastatic malignancy has resulted in cases of DIPNECH coming to light which would otherwise have gone undetected. This is considered more likely than an increased risk of DIPNECH in association with malignancy at other sites.

Our data also show that DIPNECH is typically an indolent and non-progressive disorder, with $83 \%$ of patients with symptoms being alive at 5 years. However there were two patients with progressive disease, one of whom underwent transplantation for obliterative bronchiolitis ${ }^{12}$ and one who deteriorated over 4 years despite inhaled steroid therapy. The death of the patient 5 years after transplantation was considered to be due to obliterative bronchiolitis secondary to chronic rejection rather than recurrence of DIPNECH, although no autopsy was performed. However, more importantly, a "wait and watch" policy in four patients showed that the disease remained stable over many years. In the series by Aguayo et al, ${ }^{2}$ two patients underwent cycles of chemotherapy with no effect and remained stable thereafter with no treatment. Follow-up without treatment may therefore be a reasonable first course of action. However, a trial of steroid therapy seems to be reasonable if pulmonary function indices deteriorate; this was based primarily on the presence of associated mild chronic bronchiolitis in all biopsies in this series. The reason why DIPNECH is associated with obliterative bronchiolitis is uncertain, but neuroendocrine cells are known to produce potentially fibrogenic cytokines such as bombesin ${ }^{13}$ and the presence of associated inflammation may also be a contributory factor.

The cohort contains a patient with MEN type l syndrome, which is the first example to our knowledge of its association with DIPNECH, although there is one report of DIPNECH associated with acromegaly due to a pituitary adenoma, suggested as representing the extreme end of the spectrum of
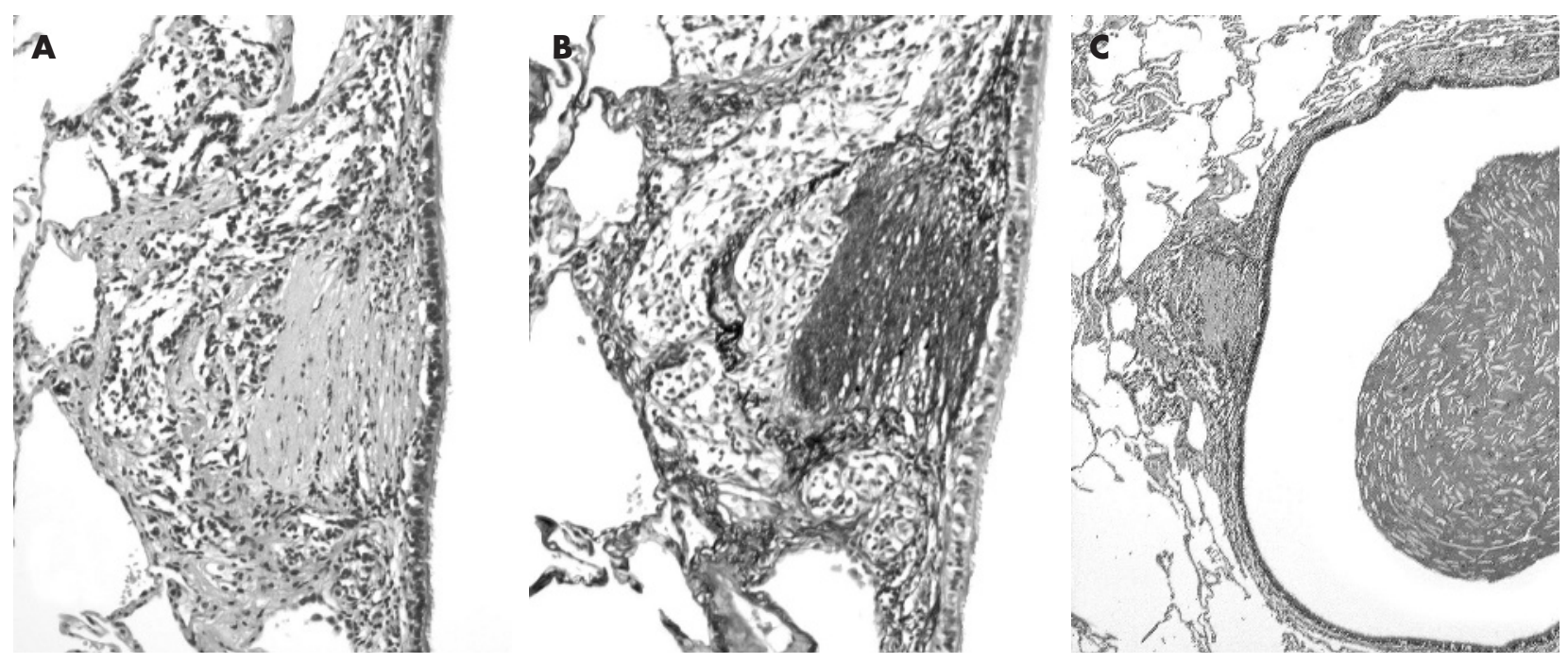

Figure 3 (A and B) Constrictive obliterative bronchiolitis in a case of diffuse idiopathic neuroendocrine cell hyperplasia (B - elastic van Gieson staining). (C) Proximal bronchiole showing bronchiolectasis and mucus plugging. 
Table 3 Histopathological features in cases of diffuse idiopathic pulmonary neuroendocrine cell hyperplasia

\begin{tabular}{llll}
\hline Histopathological features & Group 1 & Group 2 & Total \\
\hline Neuroendocrine cell hyperplasia 9/9 & $10 / 10$ & $19 / 19$ \\
Tumourlets & $9 / 9$ & $10 / 10$ & $19 / 19$ \\
Typical carcinoid & $4 / 9$ & $5 / 10$ & $9 / 19$ \\
Atypical carcinoid & $0 / 9$ & $3 / 10$ & $3 / 19$ \\
Bronchiolitis & $9 / 9$ & $10 / 10$ & $19 / 19$ \\
Obliterative bronchiolitis & $7 / 9$ & $7 / 10$ & $14 / 19$ \\
Peribronchial fibrosis & $6 / 9$ & $8 / 10$ & $14 / 19$ \\
Bronchiolectasis & $4 / 9$ & $1 / 10$ & $5 / 19$ \\
Mucus plugging & $5 / 9$ & $4 / 10$ & $9 / 19$ \\
TTF-1 staining of NEH/TL cells & $5 / 5$ & $6 / 6$ & $11 / 11$ \\
THF-1 staining of TC & N/A & $3 / 3$ & $3 / 3$ \\
TTF-1 staining of AC & N/A & $2 / 2$ & $2 / 2$ \\
\hline
\end{tabular}

$A C$, atypical carcinoid; $N E$, neuroendocrine; $N E H$, neuroendocrine cell hyperplasia; TC, typical carcinoid; TL, tumourlet; TF-1, thyroid transcription factor-1.

MEN type 1 syndrome. ${ }^{14}$ This cohort also contains the first three cases of DIPNECH associated with atypical carcinoids, one being the case associated with MEN 1 syndrome and one isolated case with $\mathrm{N} 2$ disease. This indicates that not all lesions should be regarded as benign or low-grade in the setting of DIPNECH, and therefore has important management implications for those patients presenting with nodules.

Our data also provide further insights into the histology of DIPNECH. The association between peripheral carcinoids and neuroendocrine cell hyperplasia is well described ${ }^{9}$ but we also noted that many of the carcinoids had a prominent spindle cell component, a feature that seems to be more common in the rarer peripheral carcinoids than those that present in a more central location. ${ }^{15}$ Histological features did not differ noticeably between groups 1 and 2, except that tumours were more frequent in patients who were asymptomatic. We also identified associated chronic inflammation, increased airway wall thickening and constrictive obliterative bronchiolitis, the last feature even in patients with normal lung function. Indeed, given the limitations of sampling in a retrospective study such as this, these features would probably have been more often identified if more tissue had been assessed at the time of operation. Bronchiolectasis and mucus plugging are likely to be secondary to the constrictive obliterative bronchiolitis in these biopsies and, although neuroendocrine cell hyperplasia is known to be a secondary phenomenon in patients with established bronchiectasis, ${ }^{1}$ this was not thought to be the case in any of our patients.

Finally, we undertook staining for TTF-1 in 11 cases and found weak positive staining in all cases, both in carcinoids and tumourlets. There has been some controversy as to whether the low-grade pulmonary neuroendocrine tumours stain with this antibody, although some now accept that a percentage of pulmonary carcinoids, especially those that are peripheral in location, will show weak positive staining. ${ }^{15} 16$ This study additionally shows that similar low-intensity staining can be seen in the preneoplastic lesions.

We recognise that this study has some drawbacks. We accept that subdivision of certain cases is subjective-for example, one case with dyspnoea had imaging for an acute exacerbation with identification of a coin lesion but we chose to place this in group 1 rather than group 2 . We also took the view that haemoptysis was not due to DIPNECH in one patient after the longitudinal data in the case history were reviewed. In addition, no expiratory HRCT scans were undertaken, which may have increased the frequency with which mosaicism was seen. Finally, as this is a retrospective review, data are focally incomplete and have been drawn from multiple institutions where lung function results would have been collected under different conditions. Nevertheless, we believe that our conclusions drawn from the collected information are robust.

In summary, DIPNECH seems to be more common than was previously thought and may be associated with atypical carcinoids. This increased recognition is partly the result of the increased usage and accuracy of investigative imaging. Independent of presentation, most cases remain stable over many years although a few patients progress to severe airflow obstruction.

\section{ACKNOWLEDGEMENTS}

We thank the following people for taking the time to provide clinical data for this study: Dr G van Pittius, University Hospital of North Staffordshire; Dr F Black, Royal Victoria Hospital, Newcastle; Dr D Jackson, York Hospitals NHS Trust; Professor P Goldstraw, Royal Brompton Hospital; Mr G Ladas, Royal Brompton Hospital.

\section{Authors' affiliations}

Susan J Davies, Margaret M Burke, Mary N Sheppard, Andrew

G Nicholson, Department of Histopathology, Royal Brompton and Harefield Hospitals NHS Trust, London, UK

David M Hansell, Department of Radiology, Royal Brompton and Harefield Hospitals NHS Trust, London, UK

Athol U Wells, Roland M du Bois, Department of Respiratory Medicine, Royal Brompton and Harefield Hospitals NHS Trust, London, UK John R Gosney, Department of Histopathology, Royal Liverpool University Hospital, Liverpool, UK

Competing interests: None declared.

\section{REFERENCES}

1 Gould VE, Lee I, Warren WH. Immunohistochemical evaluation of neuroendocrine cells and neoplasms of the lung. Pathol Res Pract 1988;183:200-13.

2 Aguayo SM, Miller YE, Waldron JA Jr, et al. Brief report: idiopathic diffuse hyperplasia of pulmonary neuroendocrine cells and airways disease. NEngl JMed 1992;327:1285-8.

3 Felton WL, Liebow AA, Lindskog GE. Peripheral and multiple bronchial adenomas. Cancer 1953:6:555-66.

4 Lee JS, Brown KK, Cool C, et al. Diffuse pulmonary neuroendocrine cell hyperplasia: radiologic and clinical features. J Comput Assist Tomogr 2002;26:180-4.

5 Armas OA, White DA, Erlandson RA, et al. Diffuse idiopathic pulmonary neuroendocrine cell proliferation presenting as interstitial lung disease. Am J Surg Pathol 1995; 19:963-70.

6 Carmichael MG, Zacher LL. The demonstration of pulmonary neuroendocrine cell hyperplasia with tumorlets in a patient with chronic cough and a history of multiple medical problems. Mil Med 2005:170:439-41.

7 Adams H, Brack T, Kestenholz P, et al. Diffuse idiopathic neuroendocrine cell hyperplasia causing severe airway obstruction in a patient with a carcinoid tumor. Respiration 2006;73:690-3

8 Quanjer PH. Standardised lung function testing. Clin Respir Physiol 1983;19(Suppl): 1-95.

9 Travis WD, Brambilla E, Muller-Hermelink H-K, et al, eds. WHO classification:pathology and genetics.Tumours of the lung, pleura, thymus and heart. Lyons: IARC Press, 2004.

10 Gould VE, Linnoila RI, Memoli VA, et al. Neuroendocrine components of the bronchopulmonary tract: hyperplasias, dysplasias, and neoplasms. Lab Invest 1983;49:519-37

11 Miller RR, Muller NL. Neuroendocrine cell hyperplasia and obliterative bronchiolitis in patients with peripheral carcinoid tumors. Am J Surg Pathol 1995; 19:653-8.

12 Sheerin N, Harrison NK, Sheppard MN, et al. Obliterative bronchiolitis caused by multiple tumourlets and microcarcinoids successfully treated by single lung transplantation. Thorax 1995;50:207-9.

13 Aguayo SM, King TE Jr, Waldron JA Jr, et al. Increased pulmonary neuroendocrine cells with bombesin-like immunoreactivity in adult patients with eosinophilic granuloma. J Clin Invest 1990;86:838-44.

14 Fessler MB, Cool CD, Miller YE, et al. Idiopathic diffuse hyperplasia of pulmonary neuroendocrine cells in a patient with acromegaly. Respirology 2004:9:274-7.

15 Du E, Goldstraw P, Zacharias J, et al. TTF-1 expression in tumorlets, typical and atypical carcinoids, and large cell neuroendocrine carcinomas. Hum Pathol 2004;35:825-31

16 Oliveira AM, Tazelaar HD, Myers JL, et al. Thyroid transcription factor-1 distinguishes metastatic pulmonary from well-differentiated neuroendocrine tumors of other sites. Am J Surg Pathol $2001 ; 25: 815-9$. 